\title{
The third release of the fourth Fermi LAT source catalog
}

\section{B. Lott ${ }^{a, *}$, J. Ballet ${ }^{b}$, P. Bruel ${ }^{c}$, and T. Burnett ${ }^{d}$ for the Fermi-LAT collaboration}

${ }^{a}$ Centre d'Études Nucléaires de Bordeaux Gradignan, IN2P3/CNRS, Université Bordeaux, BP120, F-33175 Gradignan Cedex, France

${ }^{b}$ AIM, CEA, CNRS, Université Paris-Saclay, Université Paris Diderot, Sorbonne Paris Cité, F-91191 Gif-sur-Yvette, France

${ }^{c}$ Laboratoire Leprince-Ringuet, École polytechnique, CNRS/IN2P3, F-91128 Palaiseau, France

${ }^{d}$ Department of Physics, University of Washington, Seattle, WA 98195-1560, USA

E-mail: lott@cenbg.in2p3.fr

The third release of the fourth general catalog of Fermi-LAT sources (4FGL-DR3), based on 12 years of data between $50 \mathrm{MeV}$ and $1 \mathrm{TeV}$, is presented. Improvements in the analysis method relative to the original 4FGL catalog and new features are reviewed. The 4FGL-DR3 includes $\simeq 900$ more sources than the previous release (4FGL-DR2, obtained with 10 years of data) and $\simeq 1500$ more sources than the 4FGL. About $43 \%$ of the new sources are associated with counterparts at other wavelengths, which are mostly blazar candidates. The properties of the global set of unassociated sources reported in the catalog are discussed, with particular attention to those lying close to the Galactic plane. A population of unassociated sources that do not fit in with already known classes of gamma-ray emitters is emphasized.

$37^{\text {th }}$ International Cosmic Ray Conference (ICRC 2021)July 12th-23rd, 2021

Online - Berlin, Germany

\footnotetext{
*Presenter
} 


\section{Introduction}

Since its launch in 2008, the Fermi-LAT has revolutionized our knowledge of the gamma-ray sky. Some most spectacular manifestations of this revolution come from the detection of much larger source populations (with improved characterization) than previously achieved combined with the discovery of new classes of gamma-ray emitters. These aspects are well reflected in the general source catalogs released over the years by the LAT-collaboration. The fourth catalog of LAT detected sources, 4FGL [1], was established with 8 years of data and included 5065 sources. It will be referred to as 4FGL-DR1 in the following. Updates to this catalog enabled by the accumulation of two extra years of data are periodically released. Updates use the same data version and Galactic diffusion emission model as the initial catalog. Sources previously reported are kept in (even if they fall below the $\mathrm{TS}=25$ threshold over the extended period of data taking) with their original positions, in contrast to new catalogs where all positions are reevaluated and subthreshold sources are omitted.

A first update (4FGL-DR2, 10 years of data) was made public in 2020 with 723 new sources [2]. In this talk, we present the third release of the catalog (4FGL-DR3) based on a total of 12 years of data and including 893 new sources (all tallies are still preliminary), with a median energy-flux limit of $0.9 \mathrm{eV} \mathrm{cm}^{-2} \mathrm{~s}^{-1}$. We also discuss the properties of the unassociated sources in some detail.

\section{Analysis improvements}

The methodology that was followed is essentially the same as in the 4FGL-DR1 catalog. The first stage includes the detection and localization of the sources. The second one comprises the thresholding step, the spectral characterization and the production of light curves. The same flags as in 4FGL-DR1, indicating the limited robustness of the results against the variation of different analysis ingredients or warning about particular source conditions (e.g., proximity to a bright source), are generated. The associations with counterparts known at other wavelengths constitute the final stage of the analysis.

The (non-exhaustive) list of changes relative to 4FGL-DR2 is as follows. An updated version of the instrument-response functions (P8R3_SOURCE_V3) has been used. The weights, downplaying the contribution of low-energy, low-latitude photons to the likelihood in order to reduce the associated systematics have been recalculated. The handling of the energy dispersion has been improved. Bayesian priors have been applied when fitting the parameters of the diffuse emission model in each region of interest to hinder their excursion relative to their expected values (normalization $=1$, spectral-index correction $=0$ ). Three more extended sources than in 4FGL-DR2 have been considered, while four others have been updated. A new spectral functional form has been used for pulsars to reduce the inter-parameter correlation. The threshold for considering spectral curvature as significant has been lowered from $3 \sigma$ to $2 \sigma$, leading to an increase of the fraction of curved sources from $30 \%$ in 4FGL-DR1 to 54\% in 4FGL-DR3. This change has removed unphysical upturns in the global source spectrum at low and high energy. The number of bins in the spectral-energy distributions has been increased from 7 to 8 . The yearly light curves have been updated (while the two-monthly ones reported in 4FGL-DR1 have not). 
Table 1: LAT 4FGL-DR3 Source Classes

\begin{tabular}{|c|c|c|c|c|}
\hline \multirow[t]{2}{*}{ Description } & \multicolumn{2}{|c|}{ Identified } & \multicolumn{2}{|c|}{ Associated } \\
\hline & Designator & Number & Designator & Number \\
\hline Young pulsars, identified by pulsations & PSR & 141 & $\ldots$ & $\ldots$ \\
\hline Young pulsars, no pulsations seen in LAT yet & $\ldots$ & $\ldots$ & psr & 2 \\
\hline Millisecond pulsars, identified by pulsations & MSP & 114 & $\ldots$ & $\ldots$ \\
\hline Millisecond pulsars, no pulsations seen in LAT yet & $\ldots$ & $\ldots$ & msp & 33 \\
\hline Pulsar wind nebula & PWN & 11 & pwn & 8 \\
\hline Supernova remnant & SNR & 24 & snr & 18 \\
\hline Supernova remnant / Pulsar wind nebula & SPP & 0 & spp & 114 \\
\hline Globular cluster & GLC & 0 & glc & 34 \\
\hline Star-forming region & SFR & 3 & sfr & 2 \\
\hline High-mass binary & HMB & 8 & $\mathrm{hmb}$ & 3 \\
\hline Low-mass binary & LMB & 2 & $\operatorname{lmb}$ & 4 \\
\hline Binary & BIN & 1 & bin & 8 \\
\hline Nova & NOV & 3 & nov & 0 \\
\hline BL Lac type of blazar & BLL & 22 & bll & 1328 \\
\hline FSRQ type of blazar & FSRQ & 43 & fsrq & 734 \\
\hline Radio galaxy & RDG & 6 & $\operatorname{rdg}$ & 38 \\
\hline Non-blazar active galaxy & AGN & 1 & agn & 10 \\
\hline Steep spectrum radio quasar & SSRQ & 0 & ssrq & 2 \\
\hline Compact Steep Spectrum radio source & CSS & 0 & $\operatorname{css}$ & 5 \\
\hline Blazar candidate of uncertain type & $\mathrm{BCU}$ & 2 & bcu & 1612 \\
\hline Narrow-line Seyfert 1 & NLSY1 & 4 & nlsy1 & 4 \\
\hline Seyfert galaxy & SEY & 0 & sey & 2 \\
\hline Starburst galaxy & SBG & 0 & $\operatorname{sbg}$ & 8 \\
\hline Normal galaxy (or part) & GAL & 2 & gal & 5 \\
\hline Unknown & UNK & 0 & unk & 137 \\
\hline Total & $\ldots$ & 387 & $\ldots$ & 4111 \\
\hline Unassociated & $\ldots$ & $\ldots$ & $\ldots$ & 2160 \\
\hline
\end{tabular}

Note: The designation 'spp' indicates potential association with SNR or PWN. Designations shown in capital letters are firm identifications; lower case letters indicate associations.

\section{Source associations}

The association procedure makes use of two different methods, the Bayesian method [3] and the likelihood-ratio method [4, 5], which are both based on spatial coincidence. Only associations with a probability greater than 0.8 of being real are retained. The overall association summary for the different classes is presented in Table 1. Since the 4FGL release, gamma-ray pulsation has been detected in 13 more 4FGL-DR1 sources.

Associations have been obtained for 385 DR3 sources, representing an association fraction of $43 \%$ (down from $47 \%$ for DR2). This fraction is roughly consistent with that expected for the low-TS sources making up the new DR3 sample, based on DR1. These associations comprise:

- four pulsars with detected pulsations, including three young pulsars PSR J0922+0638, PSR J1731-4744, PSR J1831-0952, and one MSP, PSR J1455-3330.

- two MSP candidates, PSR J1957+2516 and PSR J2051+50;

- 308 blazar candidates including 233 of unknown type (BCUs), 41 BL Lacs, 34 FSRQs; 

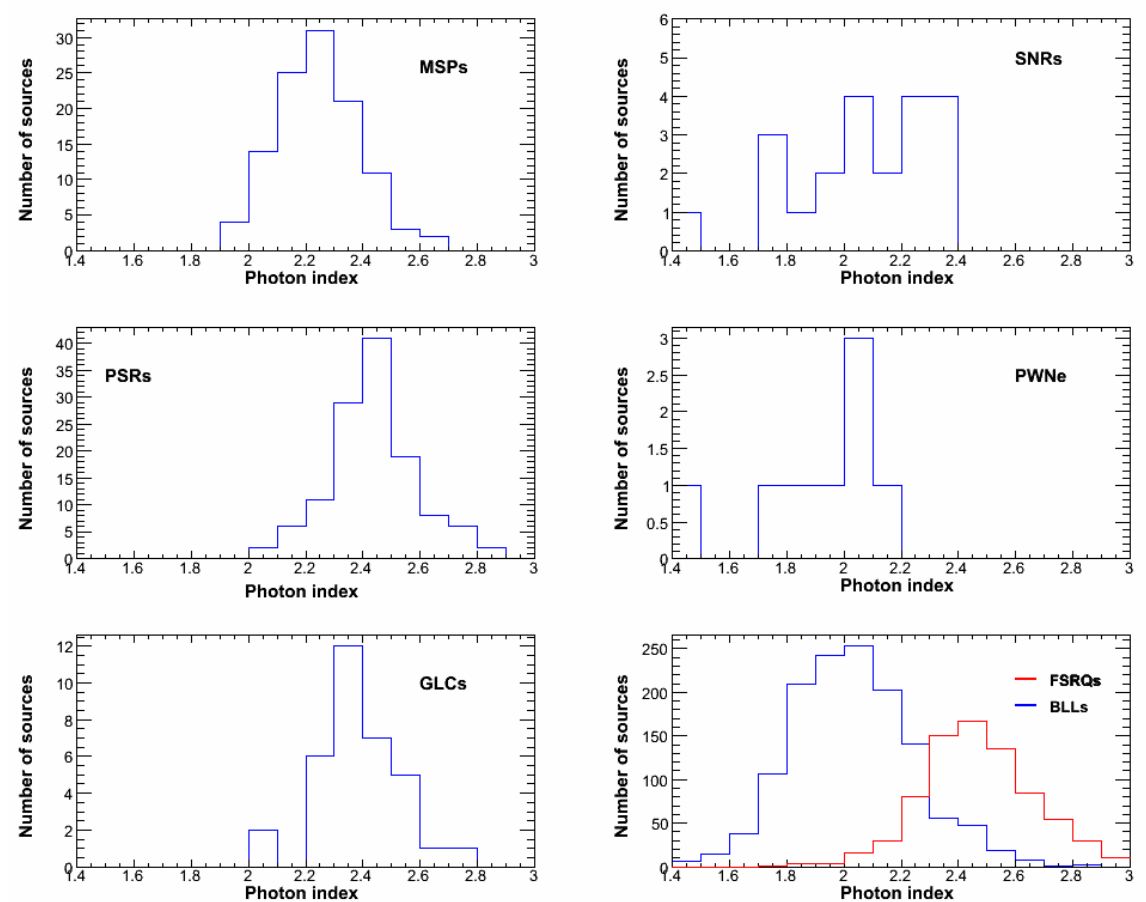

Figure 1: Photon-index distributions for different source classes.

- four globular clusters NGC 1851, NGC 5286, NGC 6205, NGC 6712;

- two pulsar wind nebulae, PWN G327.1-1.1 and PWN G54.1+0.3 ;

- 21 SPPs (sources of unknown nature but overlapping with known supernova remnants or pulsar-wind nebulae and thus candidates to these classes);

- 21 sources in radio or X-ray surveys of unknown nature.

In the following, we will consider the whole sample of sources included in the DR3 release. Fig. 1 compares the photon-index $(\Gamma)$ distributions for different established source classes. Although some classes, most notably that of pulsars, show significant levels of spectral curvature, the powerlaw photon index is a convenient parameter enabling comparison across classes. Young pulsars (PSRs) show remarkably consistent soft spectra, along with globular clusters. Millisecond pulsars (MSPs) have somewhat harder spectra on average than do young pulsars and globular clusters (GLCs). The distribution for blazars spans a broad range of photon index, the distributions for FSRQs and BL Lacs exhibiting moderate overlap, as previously noted [e.g., see 6]. Blazars constitute the only abundant class showing a hard-spectrum component. These distributions will enable shedding light on the nature of the unassociated sources later on.

\section{Unassociated sources}

Unassociated sources lying at low- $\left(|b| \leq 10^{\circ}\right)$ and high-Galactic latitudes $\left(|b|>10^{\circ}\right)$ show different properties as reported in [1], justifying it to treat them separately. The two samples are 


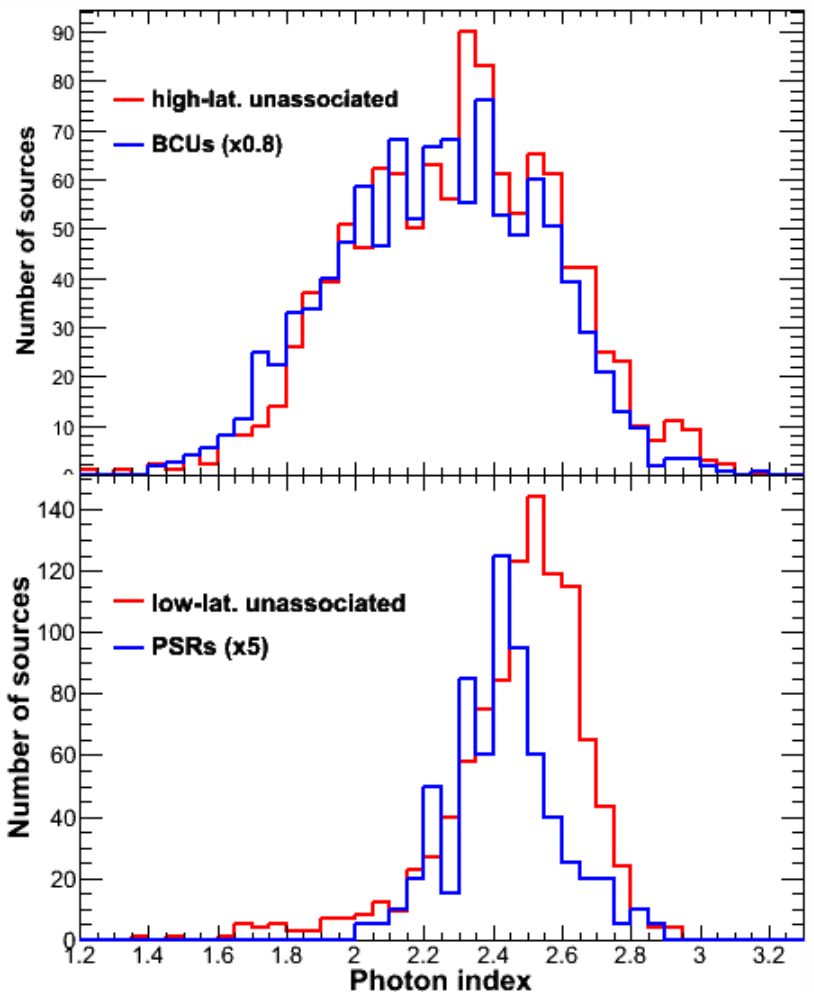

Figure 2: Top: Photon-index distribution of high-latitude $\left(|b|>10^{\circ}\right)$ unassociated sources compared to that of blazars of unknown type. Bottom: Photon-index distribution of low-latitude $\left(|b| \leq 10^{\circ}\right)$ unassociated sources compared to that of young pulsars.

about equal in size (1024 and 1129 sources below and above $|b|=10^{\circ}$ respectively). Drawing on the class-wise discriminating power of the photon index distributions illustrated in Fig. 1, the distribution for high-latitude unassociated sources is compared to that of BCUs, which is the most relevant blazar class given the low-TS range, in Fig. 2 top. The good agreement between the two distributions supports the conclusion that most high-latitude unassociated sources are indeed blazars. However, deeper investigation allows identifying some millisecond-pulsar candidates (provided their detection significance is high enough) thanks to their stronger curvature and their lack of variability relative to blazars.

We now turn to the case of low-latitude unassociated sources. Their Galactic latitude distribution (Fig. 3) shows a narrow gaussian-like component centered on the plane, and two broad shoulders extending out to about $|b|=10^{\circ}$, (comprising about $20 \%$ and $80 \%$ of the sources respectively). This distribution is quite different from those observed for other classes, most notably that of pulsars. Many sources show a notable level of clustering, forming a few broad "hot-spots" along the Galactic plane (see Fig. 4).

The photon-index distribution is compared to that of young pulsars in the lower panel of Fig. 2. The distribution is remarkably narrow and contrasts sharply with that observed at higher latitude (Fig. 2 top). It peaks around $\Gamma \simeq 2.5$, with a tail toward lower $\Gamma$ values. This tail might be due to blazars, SNRs or PWNe (Fig. 1). The high- $\Gamma$ end of the distribution is peculiar. No established 


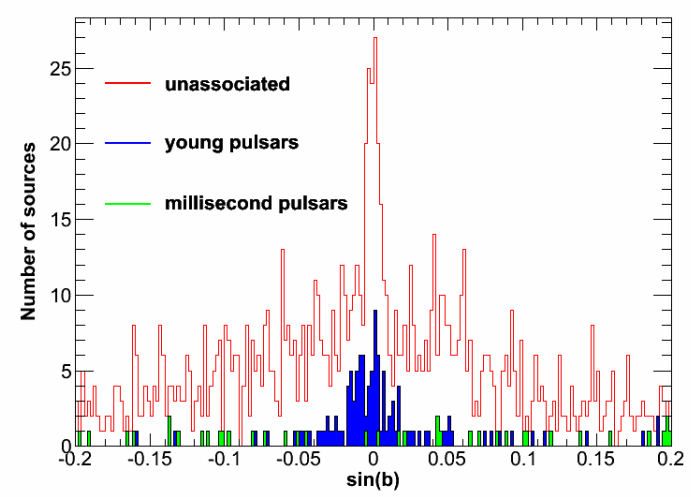

Figure 3: Galactic-latitude distribution of unassociated sources around the Galactic plane compared to that of pulsars.

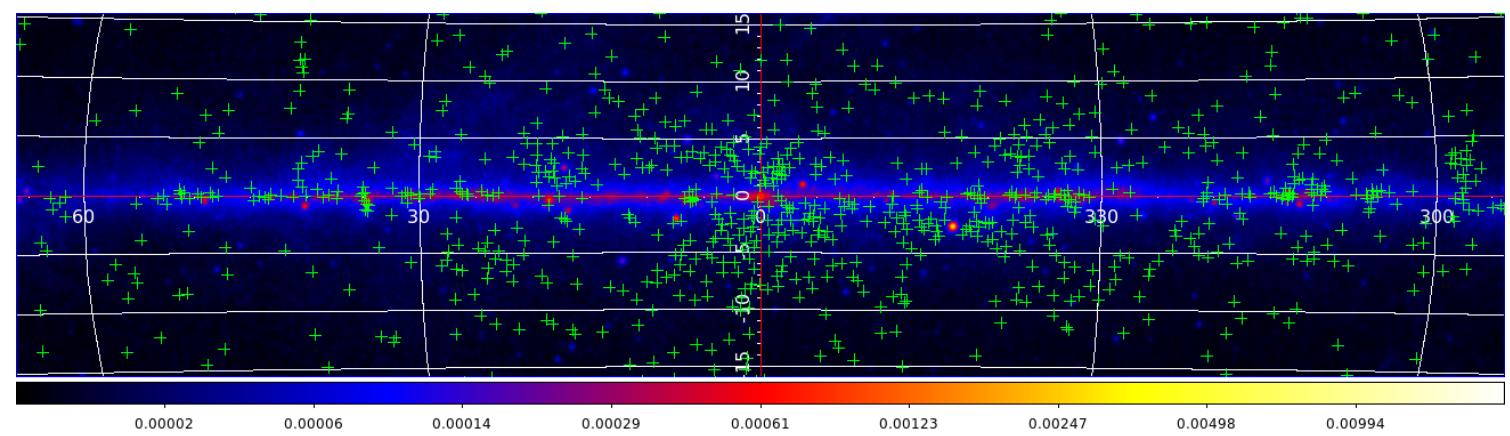

Figure 4: Positions of unassociated sources (green crosses) around the Galactic Center. The background is the Fermi-LAT intensity map above $1 \mathrm{GeV}$ in Galactic coordinates and Hammer-Aitoff projection in units of $\mathrm{cm}^{-2} \mathrm{~s}^{-1} \mathrm{sr}^{-1}$

classes of Galactic gamma-ray emitters exhibit such a soft component, not even young pulsars. The distribution is essentially constant across the plane. For strong enough sources, significant spectral curvature is detected, of the same magnitude as in the case of pulsars.

Could these sources originate from mismodeled Galactic diffuse emission? This emission is not fully understood and its modeling suffers many uncertainties. The Pass8 Galactic diffuse emission model is described in detail in [7]. To produce the model, the contributions of different components: HI, CO, Dark Neutral Medium (DNM), and Inverse Compton, whose properties are established at larger wavelengths, were fitted to the data in 9 rings of increasing Galactocentric distances. An ad-hoc component called the patch encapsulates the contributions of non-template components like the Galactic Center excess, Loop I or the Fermi bubbles. The patch was smoothed out not to absorb features with spatial scales less than $4^{\circ}$. Simulations with enhanced contributions of either $\mathrm{CO}, \mathrm{DNM}$ or the patch have been performed to investigate whether the spurious point-like sources spawned from these extra contributions share common features with the observed unassociated sources. Early results show that the thus-obtained TS $>25$ sources exhibit even softer spectra ( $\Gamma \simeq 2.7$ ) than the unassociated sources, apparently undermining this possibility. Other intriguing 
possibilities concern so-far unidentified large-scale features or the existence of an abundant, entirely new class of sources.

\section{Summary}

The 4FGL-DR3 release, based on 12 years of data and foreseen to be made public in the Summer of 2021, has been presented, with about 900 more sources than the previous 4FGL-DR2. Associations have been found for $43 \%$ of the new source, mostly with blazar candidates. The overall sample of unassociated sources shows a strong evolution as a function of Galactic latitude. While the high-latitude population is probably dominated by blazars, the nature of the bulk of the sources detected at lower latitudes remains unclear as neither their latitude distribution nor their spectral softness fit in with any known classes of gamma-ray emitters.

\section{Acknowledgements}

The Fermi-LAT Collaboration acknowledges support for LAT development, operation and data analysis from NASA and DOE (United States), CEA/Irfu and IN2P3/CNRS (France), ASI and INFN (Italy), MEXT, KEK, and JAXA (Japan), and the K.A. Wallenberg Foundation, the Swedish Research Council and the National Space Board (Sweden). Science analysis support in the operations phase from INAF (Italy) and CNES (France) is also gratefully acknowledged. This work performed in part under DOE Contract DE-AC02-76SF00515.

\section{References}

[1] Abdollahi, S., et al. 2020, ApJS, 247, 33 (4FGL)

[2] The Fermi-LAT collaboration. 2020, arXiv:2005.11208 (4FGL-DR2)

[3] Abdo, A., et al., 2010, ApJS, 188, 405 (1FGL)

[4] Ackermann, M., et al., 2011, ApJ, 743, 171 (2LAC)

[5] Ackermann, M. et al. 2015, ApJ, 810, 14 (3LAC)

[6] Ajello, M. et al. 2020, ApJ, 892, 105 (4LAC)

[7] https://fermi.gsfc.nasa.gov/ssc/data/analysis/software/aux/4fgl/Galactic_Diffuse_Emission_Model_for_the_4FGL_Catalog_Analysis.pdf (Diffuse emission model) 